\title{
CONVEX COMBINATIONS OF UNIMODULAR FUNCTIONS
}

\author{
BY WALTER RUDIN ${ }^{1}$
}

Communicated by M. H. Protter, February 20, 1969

Stephen Fisher has recently proved [1] that the set of all convex combinations of finite Blaschke products is dense in the unit ball of the disc algebra. The restriction of the disc algebra to the unit circle $T$ is the subalgebra $A$ of $C(T)$ which consists of those $f \in C(T)$ whose Fourier transforms are supported by the semigroup of the nonnegative integers. The unimodular members of $A$ (i.e., those $f \in A$ for which $|f|=1$ on $T$ ) are the (restrictions to $T$ of the) finite Blaschke products. Hence Fisher's result is a special case of the following

Theorem. Let $\Gamma$ be the dual of a compact abelian group $G$, let $\Sigma$ be a semigroup in $\Gamma$, let $C_{\Sigma}$ consist of all $f \in C(G)$ whose Fourier transforms $\hat{f}$ are supported by $\Sigma$. Then the set of all finite convex combinations of unimodular members of $C_{\Sigma}$ is dense (relative to the supremum norm) in the unit ball of $C_{\Sigma}$.

The proof is based on the three lemmas stated below. Lemmas 1 and 2 are of some interest even in the classical case $G=T$. Lemma 3 is a technicality; it is used in the proof of Lemma 2. Terminology and notation are as in [3]. In particular, the * in Lemma 1 denotes convolution, and $M(G)$ is the set of all complex Borel measures on $G$.

Lemma 1. Suppose $Q$ is a closed, convex, balanced subset of $C(G)$ which is translation-invariant. Suppose $f \in C(G)$ but $f \notin Q$. Then there exists $\mu \in M(G)$ such that

(i) $(f * \mu)(0)>1$,

(ii) $\|g * \mu\|_{\infty}<1$ for every $g \in Q$,

(iii) $\hat{\mu}$ has finite support in $\Gamma$.

Lemma 2. Suppose $\Sigma$ is a semigroup in $\Gamma, \Sigma$ is not a group, $f \in C_{\Sigma}$, $\|f\|_{\infty}<1$, and $E$ is a finite subset of $\Gamma$. Then there exists a unimodular $g \in C_{\Sigma}$ such that $\hat{g}(\gamma)=\hat{f}(\gamma)$ for every $\gamma \in E$.

LEMMA 3. If $\Lambda$ is a finitely generated abelian group and if $S$ is a semigroup in $\Lambda$ which is not a group, then there is a homomorphism $\phi$ of $\Lambda$ into the real line such that $\phi(s) \geqq 0$ for every $s \in S$ and $\phi\left(s_{0}\right)=1$ for some $s_{0} \in S$.

The theorem is an easy consequence of Lemmas 1 and 2. First, if $\Sigma$ is a group, then $C_{\boldsymbol{Z}}$ is the same as $C(H)$ where $H$ is the quotient of

1 Partially supported by NSF Grant GP-6764. 
$G$ modulo the annihilator of $\Lambda$, and the conclusion of the theorem is known to hold in $C(X)$ for every compact Hausdorff space $X$ [2, Theorem 1]. So assume $\Sigma$ is not a group, pick $f \in C_{\Sigma}$ with $\|f\|_{\infty}<1$, pick $\mu \in M(G)$ so that $\hat{\mu}$ has finite support $E$. By Lemma 2 there is a unimodular $g \in C_{\Sigma}$ such that $f * \mu=g * \mu$. Let $Q$ be the closed convex hull of the unimodular functions in $C_{\Sigma}$. This $Q$ satisfies the hypotheses of Lemma 1. Since $f * \mu=g * \mu$, Lemma 1 implies that $f \in Q$, which proves the theorem.

We now sketch the proofs of the lemmas.

Proof of Lemma 1. Assume, without loss of generality, that $f$ is a trigonometric polynomial on $G$. By the Hahn-Banach separation theorem [5, p. 108, Theorem 3] there exists $\delta>0$ and $\nu \in M(G)$ such that $(f * \nu)(0)>1$ but $|(g * \nu)(0)|<1-\delta$ for every $g \in Q$. Since $Q$ is invariant under translations of $G$, the last inequality holds for all translates of $g$, so $\|g * \nu\|_{\infty}<1-\delta$. There is a trigonometric polynomial $P$ such that $\hat{P}=1$ on the support of $\hat{f}$ and $\|P\|_{1}<1+\delta[3$, Theorem 2.6.8]. Define $\mu=P * \nu$. Then $\operatorname{supp} \hat{\mu} \subset \operatorname{supp} \hat{P}, f * \mu=f * \nu$, and for every $g \in Q$,

$$
\|g * \mu\|_{\infty}=\|g * \nu * P\|_{\infty} \leqq\|g * \nu\|_{\infty}\|P\|_{1}<1-\delta^{2} .
$$

Proof of Lemma 2. There is a trigonometric polynomial $P \in C_{\Sigma}$ such that $\|P\|_{\infty}<1$ and $\hat{P}=\hat{f}$ on $E$. Let $\left\{\gamma_{1}, \cdots, \gamma_{k}\right\}$ be the support of $\hat{P}$, choose $\gamma_{0} \in \Sigma$ so that $-\gamma_{0} \notin \Sigma$, let $\Lambda$ be the subgroup of $\Gamma$ generated by $E$ and $\gamma_{0}, \gamma_{1}, \cdots, \gamma_{k}$, put $S=\Lambda \cap \Sigma$, choose $\phi$ and $s_{0}$ as in Lemma 3. Since $E$ is finite, there is a positive integer $N$ such that $N>\phi(\gamma)$ for every $\gamma \in E$. Define

$$
\Psi(x)=\left(x, N s_{0}+\gamma_{1}+\cdots+\gamma_{k}\right) \quad(x \in G) .
$$

Then $\Psi \in C_{S}$ and $\Psi \bar{P} \in C_{S}$. Define

$$
g=(P-\Psi) /(1-\Psi \bar{P}) .
$$

Then $|g|=1$ on $G, g \in C_{S} \subset C_{\Sigma}$, and

$$
g-P=-\Psi+\sum_{n=1}^{\infty}(\Psi \bar{P})^{n}(P-\Psi) .
$$

If $\gamma^{\prime} \in \Lambda$ is such that some term on the right has its Fourier coefficient $\neq 0$ at $\gamma^{\prime}$, our choice of $\Psi$ implies that $\phi\left(\gamma^{\prime}\right) \geqq N>\phi(\gamma)$ for every $\gamma \in E$. Thus $\gamma^{\prime} \notin E$, and $\hat{g}=\hat{P}$ on $E$.

Proof of Lemma 3. Assume $O \in S$, without loss of generality. $\Lambda$ is (isomorphic to) the direct sum of some $Z^{n}$ and a finite abelian group $F$. For $(p, f) \in \Lambda$, where $p \in Z^{n}, f \in F$, define $\pi(p, f)=p$, and put 
$S_{0}=\pi S$. Then $S_{0}$ is a semigroup in $Z^{n}$. I claim that $S_{0}$ is not a group. For otherwise there would correspond to each $x=(p, f) \in S$ an element $y=\left(-p, f^{\prime}\right) \in S$; if $a, b$ are the orders of $f, f^{\prime}$ and if $z=(a b-1) x+a b y$, then $z \in S$ and $z+x=0$. So $S$ would be a group, contradicting the hypothesis.

We may assume that $n$ is so small that $S_{0}$ lies in no subgroup of $Z^{n}$ whose rank is less than $n$. Regard $Z^{n}$ as the subset of euclidean space $R^{n}$ whose points have only integer coordinates. Let $H$ be the smallest convex set in $R^{n}$ which contains $S_{0}$. Choose $p \in S_{0}$ such that $-p \notin S_{0}$. If $-p$ were in $H$, one can see that $-p$ would be representable as a convex combination of points of $S_{0}$, with rational coefficients, so that some positive integral multiple of $-p$ would be in $S_{0}$; the semigroup property of $S_{0}$ then implies that $-p \in S_{0}$, a contradiction. Thus $H \neq R^{n}$.

Since $H$ is a semigroup it follows that $H$ contains no neighborhood of $O$. Hence $O$ is a boundary point of $H$, and there is a hyperplane $I$ in $R^{n}$ such that $O \in I I$ and $H$ lies in one of the two closed half-spaces determined by $\Pi$. Our choice of $n$ shows that $S_{0}$ is not a subset of $\Pi$. Hence there is a linear functional $\phi_{1}$ on $R^{n}$ which has $\Pi$ for its nullspace and which is 1 at some point of $S_{0}$. Then $\phi=\phi_{1} \pi$ has the desired properties.

REMARK. With $G=T^{n}$ and $\Sigma$ the positive cone in $Z^{n}$, the theorem extends Fisher's result to the polydisc algebras [4].

\section{REFERENCES}

1. Stephen Fisher, The convex hull of the finite Blaschke products, Bull. Amer. Math. Soc. 74 (1968), 1128-1129.

2. R. R. Phelps, Extreme points in function algebras, Duke Math. J. 32 (1965), 267278.

3. Walter Rudin, Fourier analysis on groups, Interscience, New York, 1962.

4. —- Function theory in polydiscs, Benjamin, New York, 1969.

5. Kosaku Yosida, Functional analysis, Die Grundlehren der Math. Wissenschaften, Band 123, Academic Press, New York and Springer-Verlag, Berlin, 1965.

UNiversity OF Wisconsin, Madison, Wisconsin 53706 